\title{
Rescue of an Enterotropic Newcastle Disease Virus Strain ZM10 From Cloned cDNA and Stable Expressing an Inserted Foreign Gene
}

Lei He

Henan University of Science and Technology

Hairong Wang

Henan University of Science and Technology

Zuhua Yu

Henan University of Science and Technology

Chengshui Liao

Henan University of Science and Technology

Ke Ding

Henan University of Science and Technology

Cai Zhang

Henan University of Science and Technology

Chuan Yu

Henan University of Science and Technology

Chunjie Zhang ( $\sim$ cjzhang@sina.com )

Henan University of Science and Technology

\section{Research Article}

Keywords: Newcastle disease virus, Reverse genetics system, enterotropic, Red fluorescent protein

Posted Date: December 3rd, 2021

DOI: https://doi.org/10.21203/rs.3.rs-1053109/v1

License: (c) (i) This work is licensed under a Creative Commons Attribution 4.0 International License.

Read Full License 


\section{Abstract}

Background: Newcastle disease virus (NDV) strain ZM10, a typical enterotropic avirulent vaccine strain, has been widely used in in China for chickens against Newcastle disease. To elucidate its enterotropic mechanism and develop recombiant multivalent vaccines based on it, the reverse genetics system for NDV ZM10 is an indispensable platform.

Results: A full-length cDNA clone of NDV ZM10 and three supporting plasmids were constructed using the ligation-independent cloning (LIC) method. Recombinant NDV rZM10 was successfully rescued after these plasmids were co-transfected into BHK-21 cells. Besides, the recombinant virus rZM10-RFP encoding the red fluorescent protein was generated by inserting the RFP gene into the full-length clone of NDV between the $P$ and $M$ genes. These rescued viruses were genetically and biologically identical to the parental strain and showed similar growth kinetics.

Conclusion: The recovery system of NDV ZM10 strain was established, and can be used as a foundation for research on the enterotropic mechanism and development of multivalent vaccines against viral diseases of livestock and poultry.

\section{Background}

Newcastle disease (ND) is a highly contagious avian disease affecting all species of birds that can cause tremendous socio-economic impact on the poultry industry[1]. The etiological agent of ND, Newcastle disease virus (NDV), belongs to genus Avulavirus of the Paramyxoviridae family. Strains of NDV are categorized into three pathotypes depending on the pathological severity in chickens: highly virulent (velogenic), intermediate (mesogenic) and non-virulent (lentogenic) [2, 3]. Velogenic viruses are further subdivided into viscerotropic and neurotropic based on the severe symptom types of intestinal lesions and neurologic signs, respectively[4]. The infection of chicken with velogenic NDV strains causes a severe acute disease with high morbidity and mortality, characterized by the organs impairment of neurological, gastrointestinal, reproductive, while Lentogenic and mesogenic NDV strains often cause subclinical infection with no symptom or mild respiratory signs and have been used as live attenuated vaccines worldwide [5].

NDV is a non-segmented, negative sense single stranded RNA virus and comprises one of the three genome size:15,186, 15192 and 15198 containing six genes and encoding at least six proteins, the nucleocapsid protein(NP), phosphoprotein (P), matrix protein (M), fusion protein(F), hemagglutininneuraminidase protein $(\mathrm{HN})$, and largepolymerase protein $(\mathrm{L})$ arranged in the order of $3^{\prime}$ leader-NP-P-M-F-

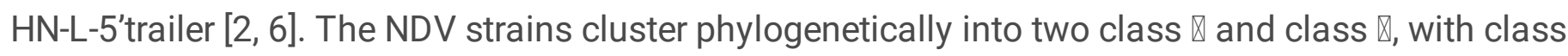
छbeing further divided into three subgenotypes and class $₫$ into 21 genotypes based on the new classification system[7]. The ZM10 strain was isolated from the intestine of broiler chickens showing no signs of respiratory disease, and replicates both in the respiratory and intestinal tract, with preference for the intestine. The ZM10 virus is a naturally attenuated lentogenic strain which has no detectable 
respiratory reaction in chickens regardless of the administration route[8,9]. It is widely used in China as a vaccine strain and could induced solid immunity in inoculated chickens with high titer NDV antibody in the serum and robust mucosal response in addition to a systemic response[8]. The enterotropic characteristics of ZM10 are extremely important for protection chickens against velogenic viscerotropic NDV which targets both intestinal and respiratory epithelia, inducing severe gross and microscopic damage.

Since the first reverse genetics system for NDV has been established in 1999[10], many NDV vaccine strains such as Anhinga LaSota[10, 11]; Beaudette C[3]; Italien[12]; Mukteswar[13] ;

Banjarmasin/010/10[14]; VG/GA[15]; TS09-C[16]; NA-1[17] and R2B [18]have been successfully rescued. The advent of the vectors of NDV strains not only allows us to study the pathogeneisis of NDV but also provides a powerful tool to develop new vaccines to express foreign genes against the diseases of poultry and other animals. It is necessary to develop the reverse genetics system for the ZM10 strain as it has been proved to be an excellent NDV vaccine candidate. Therefore, the present work describes the development of a reverse genetics system based on the full-length ZM10 cDNA. Furthermore, the recombinant virus rZM10-RFP, which expresses a reporter red fluorescent protein RFP, was also constructed as a foundation to develop it as a vaccine vector for the delivery of heterologous proteins.

\section{Methods}

\section{Viruses, Cells culture and RNA preparation}

The NDV ZM10 strain was purchased from QYH Biotech Co., Ltd and propagated in 9-day-old SPF chicken embryos. BHK-21 and DF- 1 cell lines were maintained at $37^{\circ} \mathrm{C}$ and $5 \% \mathrm{CO}_{2}$ in Dulbecco's Modified Eagle Medium (DMEM, ThermoFisher Scientific) supplemented with $10 \%$ heat-inactivated fetal calf serum (FBS, Beyotime, China), and antibiotics $(100 \mu \mathrm{g} / \mathrm{mL}$ streptomycin and $100 \mathrm{U} / \mathrm{mL}$ penicillin). The DF-1 cells were cultured in DMEM containing 10\% allantoic fluid (AF) from 10-day-old specific-pathogen-free (SPF) chicken embryos for all subsequent infection experiments unless otherwise indicated. The culture medium was replaced every 3 days.

\section{Construction of full-length NDV cDNA}

To obtain the CDNA, the original ZM10 strain was purified by the limiting dilution method through 9-dayold SPF chicken embryos for 3 passages. During each purification, the HA positive allantoic fluid of the highest dilution was collected and used for the subsequent passage. Viral genomic RNA of the purified virus was isolated with a MiniBEST Viral RNA/DNA Extraction Kit (TaKaRa Bio Inc, Japan). The full length genomic sequence of ZM10 was determined and submitted in the Genbank database (accession no. OL676769) and the Based on verified sequences of ZM10, the NDV full-length cDNA clone was constructed by three steps of the ligation-independent cloning (LIC) as illustrated in Fig. 1, by using an InFusion PCR Cloning Kit (Clontech, Mountain View, CA). Primer sets were designed to contain a 15nucleotide (nt) overlapping region of homology at their $5^{\prime}$ end to amplify the three cDNA fragments and the linearized vector backbone (Table 1). The viral genome was divided into three segments (Fragment 1 , 
Fragment 2 and Fragment 3) to construct the NDV full-length cDNA clone. In the first step, as shown in Fig. 1, Fragment 1 was generated by RT-PCR with specific primers F1 F and F1 R and cloned into the corresponding modified pBluescript vector which also linearized by PCR with specific primers using the PrimeScript $^{\text {TM }}$ high fidelity RT-PCR Kit (Takara) (F1 Vet F and F1 Vet R, Table 1), which resulted in production of a pZM10-F1 subclone. Subsequently, Fragment 2 was amplified with the primers F2 F and F2 R and cloned into the pZM10-F1 vector linearized by PCR with specific primers and F2 Vec F and F1 $R$ ), to obtain the subclone pZM10-F1-F2. Finally, The plasmid pZM10 containing the whole genome was constructed by insert the Fragment 3 into the subclone pZM10-F1-F2 using the above method with the primers F3 F and F3 R for Fragment 3 and F3 Vec F and F2 R for linearized vector pZM10-F1-F2. 
Table 1

Primer sequences of the construction of ZM10 full-length cDNA clone.

\begin{tabular}{|c|c|c|}
\hline Primer & Primer sequence & Primer name \\
\hline $1^{\mathrm{a}}$ & ACCAAACAGAGAATCCGTAAG & $\mathrm{F} 1 \mathrm{~F}$ \\
\hline $2^{a}$ & CAAGAGATATGACAGTTAAAACG & F1 R \\
\hline $3^{a}$ & CTGTCATATCTCTTGTATGTGGTATACTTAGCCTGG & $\mathrm{F} 2 \mathrm{~F}$ \\
\hline $4^{\mathrm{a}}$ & ATCCAGTCTATGTTGGAGATTCCCAGCTG & F2 R \\
\hline $5^{a}$ & CAACATAGACTGGATGACGGCATAACTCAGATGAC & F3 F \\
\hline $6^{a}$ & ACCAAACAAAGATTTGGTGAATGACAAGAC & F3 R \\
\hline $7^{b}$ & ACAACСCTGTCCTGCTTССTCTG & RFP-F \\
\hline $8^{b}$ & CGCTCGGGGTGTTGGACCTTGGG & RFP-R \\
\hline $9^{b}$ & CTGTCATATCTCTTGGGCCGGCATGGTCCCAGCCTCC & F1 Vec F \\
\hline $10^{c}$ & GATTCTCTGTTTGGTCCCTATAGTGAGTCGTATTAGCG & F1 Vec R \\
\hline $11^{c}$ & CAACATAGACTGGATGGCCGGCATGGTCCCAGCCTCC & F2 Vec F \\
\hline $12^{c}$ & AAATCTTTGTTTGGTGGCCGGCATGGTCCCAGCCTCC & F3 Vec F \\
\hline $13^{c}$ & CCAACACCCCGAGCGCAACTCTCCAAGCGGCAATC & Vec-RFP-F \\
\hline $14^{c}$ & GCAGGACAGGGTTGTAGCGAGAGAGGTAACGATTAG & Vec-RFP-R \\
\hline \multicolumn{3}{|c|}{$\begin{array}{l}\text { a Primers } 1-6 \text { were used to RT-PCR amplify the Fragment 1, Fragment 2, and Fragmen3 genes from th } \\
\text { viral RNA of NDV ZM10 strain. }\end{array}$} \\
\hline \multicolumn{3}{|c|}{ b Primers 7-8 were used to amplify the RFP genes from LS-RFP plamid. } \\
\hline \multicolumn{3}{|c|}{ c Primers 9-12 were used to amplify or linearize the subclone vectors and the vector for RFP gene. } \\
\hline
\end{tabular}


Table 2

Biological assessments of the NDV recombinant viruses.

\begin{tabular}{|llllll|}
\hline Viruses & MDT $^{\mathrm{a}}$ & ICPI $^{\mathrm{b}}$ & HA $^{\mathrm{c}}$ & EID $_{50}{ }^{\mathrm{d}}$ & TCID $_{50}{ }^{\mathrm{e}}$ \\
\hline ZM10 & $>150 \mathrm{hs}$ & 0.0 & $2^{10}$ & $3.16 \times 10^{9}$ & $1.78 \times 10^{8}$ \\
\hline rZM10 & $>150 \mathrm{hs}$ & 0.0 & $2^{10}$ & $3.16 \times 10^{9}$ & $1.78 \times 10^{8}$ \\
\hline rZM10-RFP & $>150 \mathrm{hs}$ & 0.0 & $2^{9}$ & $5.62 \times 10^{8}$ & $4.33 \times 10^{7}$ \\
\hline a Mean death time assay in embryonating eggs. \\
\hline b Intracerebral pathogenicity index assay in day-old chickens. \\
\hline c Hemagglutination assay. \\
\hline d The 50\% egg infective dose assay in embryonated eggs. \\
\hline e The 50\% tissue culture infective dose assay on DF-1 cells. \\
\hline
\end{tabular}

\section{Generation of helper plasmids of NDV strain ZM10}

Viral genomic RNA of purified ZM10 virus was isolated with a MiniBEST Viral RNA/DNA Extraction Kit (TaKaRa Bio Inc, Japan). And three cDNA fragments NP, $P$, and $L$ which contain the open reading frames (ORFs) of the nucleoprotein $(N)$, phosphoprotein $(P)$ and polymerase $(L)$ were carried out by using a highfidelity RT-PCR Reagent Kit (TaKaRa Bio Inc, Japan) with three pairs of specific primers separately. To clone the NP, $\mathrm{P}$ and $\mathrm{L}$ genes, three primer pairs of NP F (5'-AAA CAC GAT AAT ACC ATG TCT TCT GTA TTC GAT GAG-3') and NP R (5'- GTG GAT CGG ATC TTA TCA GTA CCC CCA GTC GGT GTC -3'), and P F (5'CAC GAT AAT ACC ATG GAG ATG GCC ACC TTT ACA G-3') and P R (5'-GTG GAT CGG ATC TTA TTA GCC ATT CAG TGC AAG GCG C-3'), and L F (5'and LR (5'- GTG GAT CGG ATC TTA TTA AGA GTC ACA GTT ACT GTA G -3') (Xhol, Xbal and Mlul sites are in italics and underlined) were used for PCR with PrimeSTAR® GXL DNA Polymerase (TaKaRa Bio Inc, Japan); The modified pTM vector was linearized by PCR with a pair of specific primers (Vet up TAA GAT CCG ATC CACTAG TTC TAG and Vet down GGT ATT ATC GTG TTT TTC AAA GGA AAA CC). After gel purification, the fragments NP, $P$ and $L$ gene were cloned into the linearized pTM vector using the InFusion PCR cloning kit. Corresponding plasmids were designated as pZM10-NP, pZM10-P and pZM10-L, respectively.

\section{Construction of full-length clone of NDV harbouring RFP gene}

To insert the RFP gene into the NDV full-longth clone, the RFP gene cassette was amplified by PCR from a plasmid LS-RFP by using the specific primer RFP-F and RFP-R, whereas primers Vec-RFP-F and Vec-RFP-R 
were used to amplify the linearized vector of pZM10. The RFP gene was inserted into the pZM10 between the $\mathrm{P}$ and $\mathrm{M}$ genes of the rZM10 genome to generate pZM10-RFP by using the In-Fusion PCR Cloning Kit, as shown in Fig. 1.

\section{Transfection and recovery of recombinant NDV}

To generate recombinant viruses, BHK-21 cells were grown overnight in a six-well plate to approximately $80 \%$ confluency and infected with MVA/T7 at a multiplicity of infection (MOI) of 3 before the transfection. Subsequently, the cells were co-transfected with $2 \mu \mathrm{g}$ of pTM-ZM10, $1 \mu \mathrm{g}$ of pZM10-NP, $0.5 \mu \mathrm{g}$ of pZM10-P, and $0.1 \mu \mathrm{g}$ of pZM10-L by using $6 \mu \mathrm{L}$ Lipofectamine ${ }^{\mathrm{TM}} 3000$ (Invitrogen) according to the manufacturer's instructions. After incubation for $6 \mathrm{~h}$ at $37^{\circ} \mathrm{C}$, the transfection mixture was replaced and the cells washed once by PBS buffer and maintained in Opti-MEM medium containing 10\% allantoic fluid (AF). After $72 \mathrm{~h}$, the culture supernatant of each well was harvested, clarified and injected into the allantoic cavities of 9day-old embryonated SPF eggs. 3 days later, AF was harvested and tested for NDV-specific haemagglutinating activity (HA). HA-positive viruses were filtered by $0.22 \mu \mathrm{M}$ filter twice and diluted in phosphate-buffered saline (PBS), then inoculated into chicken embryos for 3 passages. Stocks were then prepared from harvested allantoic fluids. The complete genomic sequences of the rescued viruses were determined by direct sequencing of the reverse transcription-PCR (RT-PCR) products amplified from the viral genomic RNA as described previously.

\section{Characterization of the rescued viruses}

To evaluate the biological properties of the recombinant NDV rZM10 and rZM10-RFP, the pathogenicities and growth abilities of the recombinant viruses were examined by conducting titration assays and mean death time (MDT) and intracerebral pathogenicity index (ICPI) analysis. The virus titration was measured by the standard HA test in a 96-well microplate, the $50 \%$ tissue culture infectious dose $\left(\mathrm{TCID}_{50}\right)$ assay on DF-1 cells, and the $50 \%$ egg infective dose $\left(E D_{50}\right)$ assay in 9-day-old SPF chicken embryos, and ICPI was determined in 1-day-old chickens, according to standard procedures.

To analyze the growth kinetics of the parental virus and the recombinant viruses, monolayer of DF- 1 cells (in triplicate) was infected with the each virus at $0.01 \mathrm{MOI}$, respectively. The virus lysates was harvested at 12-hour intervals, and the virus titer of each time point was measured by TCID $_{50}$ titration on DF-1 cells in triplicate from two independent experiments.

\section{Results}

\section{Construction of full-length cDNA clone of NDV strain ZM10}

The full-length clone of NDV ZM10 (pZM10) was assembled from three RT-PCR fragments which have a 15 nt overlapping region at their 5 ' end by three steps of In-Fusion PCR cloning. The genome of NDV ZM10 strain was successfully cloned into the pBluescript based vector, which was under the control of T7 promoter and flanked by HDVRz sequences and the T7 terminator sequence as illustrated in Fig. 1.A. 
These sequences ensured the cleavage at the termini and generate precise 3'and 5'viral RNA ends. The fidelity of the pZM10 plasmid was further confirmed by DNA sequencing analysis. The total length of the genome cDNA clone was 15186 bp and obeyed 'the Rule of Six' (Kolakofsky et al., 2005).

To address whether rZM10 could successfully and stably carry a foreign gene as a viral vector, the RFP gene was inserted into $p Z M 10$ between the $P$ and $M$ genes as an independent transcription unit as shown in Fig. 1.B. To generate the vector pZM10-RFP, the RFP cassette encompassed the RFP gene with the associated NDV gene-start and gene-end signals was specifically amplified from the plasmid LS-RFP with a product size of $906 \mathrm{bp}$ using PCR. And then the module was introduced in the non-coding region of the vector pZM10 between the $\mathrm{P}$ and $\mathrm{M}$ using In-Fusion ${ }^{\circledR}$ PCR cloning techniques. The plasmid were transformed into Stbl2 cells and amplified at $30^{\circ} \mathrm{C}$ for 24 hours and then purified by using a QIAprep Spin Miniprep kit (Qiagen). The total size of the p ZM10-RFP was found to be $16092 \mathrm{bp}$. The foreign RFP gene introduced into the full-length clone followed 'the rule of six'. The insert gene RFP was further verified by sequencing analysis.

\section{Rescue of recombinant NDV from cloned cDNA}

Rescue of the recombinant rZM10 or rZM10-RFP virus was performed by co-transfecting the plasmids pZM10 or pZM10-RFP respectively with the supporting plasmids pZM10-NP, pZM10-P and pZM10-L together into BHK-21 cells. The rescued viruses were amplified by inoculating $200 \mu \mathrm{L}$ of the infected cell lysate into the 9-day-old SPF chicken embryos's allantoic cavity and incubating for $96 \mathrm{~h}$ at $37^{\circ} \mathrm{C}$. Then the rescued viruses was collected by harvesting the $\mathrm{AF}$ and the virus titer was further detected by hemagglutination (HA) assay (Alexander, 1998). The rescued virus was diluted and propagated for three times in the SPF chicken embryos. The AF was harvested after the propagation and stored at $-80^{\circ} \mathrm{C}$. The insert gene and the viral genome of the rescued virus were determined by sequencing the RT-PCR products amplified from the AF. Besides, the viral cytopathic effect (CPE) produced in the BHK- 21 cells typically consisted of clumping and rounding of cells and fusion of cells with syncytia formation as observed $48 \mathrm{hpi}$ under the light microscope(Fig. 2). The expression of RFP could be observed as a bright red fluorescence under a fluorescent microscope at 200X magnifications (Nikon, Eclipse Ti, Melville, NY) (Fig. 2).

\section{Biological and molecular characterization of the recombinant viruses}

To evaluate biological properties of the parental and recombinant viruses on viral pathogenicity and growth dynamics, the recombinant NDV viruses rZM10 and rZM10-RFP were examined in vitro and in vivo by testing the virus titration (EID ${ }_{50}, T C I D 50$ and HA test), mean death time (MDT), and intracerebral pathogenicity index (ICPI) (Alexander, 1998). As shown in Table 1, the rescued viruses rZM10 and $r$ rZM10-RFP showed similar attenuated characteristics in SPF embryonated eggs and day-old chickens with a long MDT (>150h) and low ICPI (0.0) compared to the parental strain ZM10. The titers of the recombinant viruses grown in embryonated eggs were measured by $\mathrm{EID}_{50}, \mathrm{TCID}_{50}$ and $\mathrm{HA}$. The results 
showed that the recombinant virus rZM10-RFP were slightly lower but were comparable to that of rZM10 and ZM10 strain.

As shown in Fig. 3, the growth kinetics study was carried out on DF-1 cells with a collection interval of 12 $\mathrm{h}$ until $72 \mathrm{~h}$ of infection. The growth kinetics of the two recombinant viruses was slightly delayed in the onset of replication compared to the parental virus ZM10, however, after 36h, the recombinant viruses displayed a comparative replication kinetics and magnitude. The rescued virus rZM10 and rZM10-RFP had a similar kinetic and replicative efficiency as evident by the growth curve, indicating that the insertion of the RFP gene did not influence the viral replication of NDV (Fig. 3).

\section{Discussion}

Admittedly, there are a majority of infectious poultry diseases not only Newcastle disease (ND), but also other disease such as infectious bursal disease (IBD) [19, 20], infectious laryngotracheitis(ILT) [21, 22], hypervirulent fowl adenovirus (FADV) infection[23] and avian influenza (AI)[24], which endanger the poultry industry. Vaccination is a powerful and necessary mean to the controlling of these diseases. Vaccines of Newcastle disease (ND), as a compulsory immunization to most chicken farms, have been used worldwide[5]. Recombiant NDVs have been proven to be an excellent viral vector to delivery various heterogenous genes against a serial of poultry diseases including IBD, ILT, and AI[25-27]. Therefore, the development of ND as a vaccine vector may be a powerful mean to the poultry disease control. By now, several NDV strains have been rescued by reverse genetic techniques. This manipulation has facilitated the study of the molecular mechanisms of the viral pathogenicity and also provided a powerful platform for the research using NDV as vaccines vectors.

The reverse genetics system for NDV is difficult and complex compared to DNA viruses or positive-sense RNA viruses [28-30]. First of all, the naked genomic RNA of NDV is not infectious. It is only functional when being encapsidated with protein complex, ribonucleoprotein (RNP) formed by the viral NP P and L proteins. Secondly, the genome length and genome complexity have made that the cloning of the fulllength NDV genome remains the most challenging and laborious of all the steps[29, 30]. Moreover, the NDV genome has to meet particular requirements for successful rescue such as the rule-of-six[31, 32] and generation of the precise $3^{\prime}$ and $5^{\prime}$ ends[30,32]. To get the precise integral NDV genome, The traditional method is dividing the NDV genome into a set of short fragments from 6 to 11 , and it takes months even years to complete for the multiple DNA fragments cloning[10, 13,33-37]. In this study, the genome of NDV was divided into three fragments $7 \mathrm{bp}, 5 \mathrm{~kb}$ and $3 \mathrm{~kb}$. Compared to the approaches before, the design in this study needs much shorter time for the full-length genome cloning. As the amplification of a cDNA fragment around $10 \mathrm{~kb}$ is available by using the commercial high fidelity reverse transcription PCR by now, it seems the strategy of dividing the whole NDV genome into two parts is possible, which need to be explored in the future.

A majority of recombinant NDVs expressing one foreign gene (FG) as vaccines or biological therapies express through either an independent transcription unit (ITU) or an internal ribosomal entry site (IRES) 
approach $[27,35,38]$. The efficacy of these two strategies has been investigated, showing that the ITU approach was more efficient than that through the IRES approach[38]. The expression levels of FGs through ITU at different genomic locations were variable and the optimal insertion site in NDV genome for efficient expression has been identified as the non-coding region between the $P$ and $M$ genes $[15,16$, 39]. Here, the RFP gene was inserted into the NDV genome at the site of the non-recoding region between $\mathrm{P}$ and $\mathrm{M}$ gene. Our data showed that the RFP could be stably expressed in a high level in this region, and the recombinant virus rZM10-RFP did not showed any significantly differences in MDT and ICPI, indicating that the noncoding region between the $\mathrm{P}$ and $\mathrm{M}$ genes is a suitable position for $\mathrm{rZM} 10$ to express an FG and provides the design strategy for the recombinant bivalent vaccines.

Pathogens especially the viruses could infect corresponding host cell types, results in replicating in specific host organs[40-42]. The ZM10 strain has an obvious preference for replication the intestine though it could infect both the respiratory and intestinal tract. This characteristic makes the ZM10 to be an ideal vector to express the protective antigen of intestinal pathogens, therefore, an important application of our NDV rescue platform would be to use the recombinant viruses as vaccine vector for the expression of protective antigens against avian or other animals' diseases to develop multivalent vaccines.

\section{Conclusions}

In this study, the full-length genome of NDV ZM10 strain was cloned and the reverse genetic system for ZM10 was established. The recombinant ZM10 could express the RFP and both the rZM10 and rZM10RFP showed similar biological properties and growth kinetics compared to the parental virus. Considering that the ZM10 is an avirulent enterotropic NDV strain, our research provides an ideal vector for developing recombinant multivalent vaccines, especially for the pathogens grows in the intestine.

\section{Abbreviations}

ND: Newcastle disease; RFP: Red Fluorescent Protein; LIC: ligation-independent cloning; ORFs: open reading frames; $\mathrm{MOI}$ : multiplicity of infection; $\mathrm{AF}$ : allantoic fluid; $\mathrm{CPE}$ : cytopathic effect; FG: foreign gene; ITU: independent transcription unit; IRES internal ribosomal entry site.

\section{Declarations}

\section{Ethics approval and consent to participate}

All the experiments in this study were carried out in accordance with the guidelines and protocols approved by the Animal Ethics Committee of the Henan University of Science and Technology. In addition, all methods were carried out in accordance with relevant guidelines and regulations.

\section{Consent for publication}


Not applicable.

Availability of data and materials

All authors declare that the data supporting the funding of this study are available within this article.

Competing interests

The authors declare that they have no conflicts of interest.

\section{Funding}

This research was supported by the science and technology research project of Henan province (No. 192102110078) and the key project of science and technology research of Education Department of Henan Province (No. 19B230003).

\section{Authors' contributions}

LH and HW conceived and designed the experiments. ZY, CL and HW performed the molecular work, CZ and KD analyzed and interpreted all data, LH and HW analyzed wrote manuscript. CY and CZ did the study supervision. All authors read and approved the final manuscript.

\section{Acknowledgements}

The authors wish to thank HongWei Li for her excellent technical assistance and Dr QingZhong Yu for the gift of the plasmid of rLs-RFP.

\section{References}

1. Miller PJ, Decanini EL, Afonso CL: Newcastle disease: evolution of genotypes and the related diagnostic challenges. Infect Genet Evol 2010, 10(1):26-35.

2. Dimitrov KM, Lee DH, Williams-Coplin D, Olivier TL, Miller PJ, Afonso CL: Newcastle Disease Viruses Causing Recent Outbreaks Worldwide Show Unexpectedly High Genetic Similarity to Historical Virulent Isolates from the 1940s. J Clin Microbio/ 2016, 54(5):1228-1235.

3. Estevez C, King D, Seal B, Yu Q: Evaluation of Newcastle disease virus chimeras expressing the Hemagglutinin-Neuraminidase protein of velogenic strains in the context of a mesogenic recombinant virus backbone. Virus Res 2007, 129(2):182-190.

4. Seal BS, King DJ, Sellers HS: The avian response to Newcastle disease virus. Dev Comp Immunol 2000, 24(2-3):257-268.

5. Dimitrov KM, Afonso CL, Yu Q, Miller PJ: Newcastle disease vaccines-A solved problem or a continuous challenge? Vet Microbiol 2017, 206:126-136.

6. Kim SH, Samal SK: Newcastle Disease Virus as a Vaccine Vector for Development of Human and Veterinary Vaccines. Viruses 2016, 8(7). 
7. Dimitrov KM, Abolnik C, Afonso CL, Albina E, Bahl J, Berg M, Briand FX, Brown IH, Choi KS, Chvala I et al: Updated unified phylogenetic classification system and revised nomenclature for Newcastle disease virus. Infect Genet Evol 2019, 74:103917.

8. Wang LKXHBZXM: Comparative study on immunological effect of attenuated live Newcastle disease vaccine ZM10 strain. Modern Journal of Animal Husbandry and Veterinary Medicine 2008(12):38-39.

9. ; GBSLSTZQC: Comparison of respiratory tract injury between ZM10 and respiratory Newcastle disease live vaccine in chickens. China Animal Health 2014, 16(5):27-28.

10. Peeters BP, de Leeuw OS, Koch G, Gielkens AL: Rescue of Newcastle disease virus from cloned cDNA: evidence that cleavability of the fusion protein is a major determinant for virulence. $J$ Viro/ 1999, 73(6):5001-5009.

11. Romer-Oberdorfer A, Mundt E, Mebatsion T, Buchholz UJ, Mettenleiter TC: Generation of recombinant lentogenic Newcastle disease virus from cDNA. J Gen Virol 1999, 80 ( Pt 11):2987-2995.

12. Feng H, Wei D, Nan G, Cui SJ, Chen ZN, Bian H: Construction of a minigenome rescue system for Newcastle disease virus strain Italien. Arch Virol 2011, 156(4):611-616.

13. Li BY, Li XR, Lan X, Yin XP, Li ZY, Yang B, Liu JX: Rescue of Newcastle disease virus from cloned cDNA using an RNA polymerase II promoter. Arch Virol 2011, 156(6):979-986.

14. Xiao S, Nayak B, Samuel A, Paldurai A, Kanabagattebasavarajappa M, Prajitno TY, Bharoto EE, Collins PL, Samal SK: Generation by reverse genetics of an effective, stable, live-attenuated newcastle disease virus vaccine based on a currently circulating, highly virulent Indonesian strain. PLoS One 2012, 7(12):e52751.

15. Zhao W, Zhang Z, Zsak L, Yu Q: P and M gene junction is the optimal insertion site in Newcastle disease virus vaccine vector for foreign gene expression. J Gen Virol 2015, 96(Pt 1):40-45.

16. Wen G, Chen C, Guo J, Zhang Z, Shang Y, Shao H, Luo Q, Yang J, Wang H, Wang H et al: Development of a novel thermostable Newcastle disease virus vaccine vector for expression of a heterologous gene. J Gen Virol 2015, 96(Pt 6):1219-1228.

17. Sun Y, Sun M, Dai Y, Yin R, Ding Z: An improved reverse genetics system for Newcastle disease virus genotype VII. Virol Sin 2016, 31(6):521-524.

18. Chellappa MM, Dey S, Gaikwad S, Pathak DC, Vakharia VN: Rescue of a recombinant Newcastle disease virus strain R2B expressing green fluorescent protein. Virus Genes 2017, 53(3):410-417.

19. Michel LO, Kimber ML, Jackwood DJ: New introduction of a very virulent infectious bursal disease virus in New York, USA. Avian Pathol 2019, 48(5):486-491.

20. Drissi Touzani C, Fellahi S, Fassi Fihri O, Gaboun F, Khayi S, Mentag R, Lico C, Baschieri S, El Houadfi $\mathrm{M}$, Ducatez $\mathrm{M}$ : Complete genome analysis and time scale evolution of very virulent infectious bursal disease viruses isolated from recent outbreaks in Morocco. Infect Genet Evol 2020, 77:104097.

21. Gowthaman V, Kumar S, Koul M, Dave U, Murthy T, Munuswamy P, Tiwari R, Karthik K, Dhama K, Michalak I et al: Infectious laryngotracheitis: Etiology, epidemiology, pathobiology, and advances in diagnosis and control - a comprehensive review. Vet Q 2020, 40(1):140-161. 
22. Abozeid HH, Paldurai A, Varghese BP, Khattar SK, Afifi MA, Zouelfakkar S, El-Deeb AH, El-Kady MF, Samal SK: Development of a recombinant Newcastle disease virus-vectored vaccine for infectious bronchitis virus variant strains circulating in Egypt. Vet Res 2019, 50(1):12.

23. Pan Q, Yang Y, Shi Z, Liu L, Gao Y, Qi X, Liu C, Zhang Y, Cui H, Wang X: Different Dynamic Distribution in Chickens and Ducks of the Hypervirulent, Novel Genotype Fowl Adenovirus Serotype 4 Recently Emerged in China. Front Microbio/ 2017, 8:1005.

24. Li YT, Linster M, Mendenhall IH, Su YCF, Smith GJD: Avian influenza viruses in humans: lessons from past outbreaks. Br Med Bull 2019, 132(1):81-95.

25. Kim SH, Samal SK: Innovation in Newcastle Disease Virus Vectored Avian Influenza Vaccines. Viruses 2019, 11(3).

26. Dey S, Chellappa MM, Pathak DC, Gaikwad S, Yadav K, Ramakrishnan S, Vakharia VN: Newcastle Disease Virus Vectored Bivalent Vaccine against Virulent Infectious Bursal Disease and Newcastle Disease of Chickens. Vaccines (Basel) 2017, 5(4).

27. Zhao W, Spatz S, Zhang Z, Wen G, Garcia M, Zsak L, Yu Q: Newcastle disease virus (NDV) recombinants expressing infectious laryngotracheitis virus (ILTV) glycoproteins gB and gD protect chickens against ILTV and NDV challenges. J Virol 2014, 88(15):8397-8406.

28. Pfaller CK, Cattaneo R, Schnell MJ: Reverse genetics of Mononegavirales: How they work, new vaccines, and new cancer therapeutics. Virology 2015, 479-480:331-344.

29. Molouki A, Peeters B: Rescue of recombinant Newcastle disease virus: current cloning strategies and RNA polymerase provision systems. Arch Virol 2017, 162(1):1-12.

30. Molouki A, Peeters B: Rescue of recombinant Newcastle disease virus: a short history of how it all started. Arch Virol 2017, 162(7):1845-1854.

31. Peeters BP, Gruijthuijsen YK, de Leeuw OS, Gielkens AL: Genome replication of Newcastle disease virus: involvement of the rule-of-six. Arch Virol 2000, 145(9):1829-1845.

32. Kolakofsky D, Pelet T, Garcin D, Hausmann S, Curran J, Roux L: Paramyxovirus RNA synthesis and the requirement for hexamer genome length: the rule of six revisited. J Virol 1998, 72(2):891-899.

33. Krishnamurthy S, Huang Z, Samal SK: Recovery of a virulent strain of newcastle disease virus from cloned CDNA: expression of a foreign gene results in growth retardation and attenuation. Virology 2000, 278(1):168-182.

34. Yu Y, Qiu X, Xu D, Zhan Y, Meng C, Wei N, Chen H, Tan L, Yu S, Liu X et al: Rescue of virulent class I Newcastle disease virus variant 9a5b-D5C1. Virol J 2012, 9:120.

35. Zhang X, Liu H, Liu P, Peeters BP, Zhao C, Kong X: Recovery of avirulent, thermostable Newcastle disease virus strain NDV4-C from cloned cDNA and stable expression of an inserted foreign gene. Arch Virol 2013, 158(10):2115-2120.

36. Cheow PS, Tan TK, Song AA, Yusoff K, Chia SL: An improved method for the rescue of recombinant Newcastle disease virus. Biotechniques 2020, 68(2):96-100. 
37. Tavassoli A, Soleymani S, Haghparast A, Hashemi Tabar G, Bassami MR, Dehghani H: Reverse Genetics Assembly of Newcastle Disease Virus Genome Template Using Asis-Sal-Pac BioBrick Strategy. Biol Proced Online 2020, 22:9.

38. He L, Zhang Z, Yu Q: Expression of Two Foreign Genes by a Newcastle Disease Virus Vector From the Optimal Insertion Sites through a Combination of the ITU and IRES-Dependent Expression Approaches. Front Microbiol 2020, 11:769.

39. Yoshida A, Samal SK: Avian Paramyxovirus Type-3 as a Vaccine Vector: Identification of a Genome Location for High Level Expression of a Foreign Gene. Front Microbio/ 2017, 8:693.

40. Maginnis MS: Virus-Receptor Interactions: The Key to Cellular Invasion. J Mol Bio/ 2018, 430(17):2590-2611.

41. Martines RB, Ng DL, Greer PW, Rollin PE, Zaki SR: Tissue and cellular tropism, pathology and pathogenesis of Ebola and Marburg viruses. $J$ Patho/2015, 235(2):153-174.

42. Ashok A, Atwood WJ: Virus receptors and tropism. Adv Exp Med Biol 2006, 577:60-72.

\section{Figures}


A
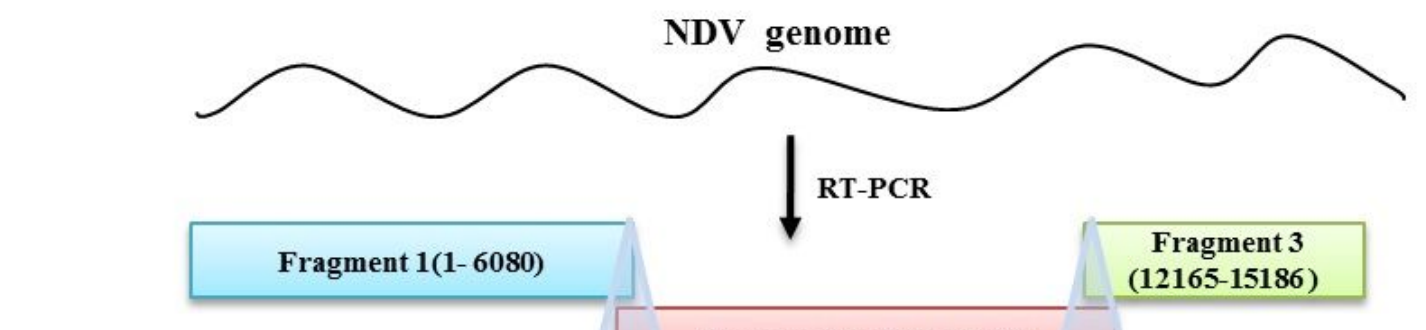

Fragment 2(6058-12193)

20 bp overlapped sequence

20 bp overlapped sequence
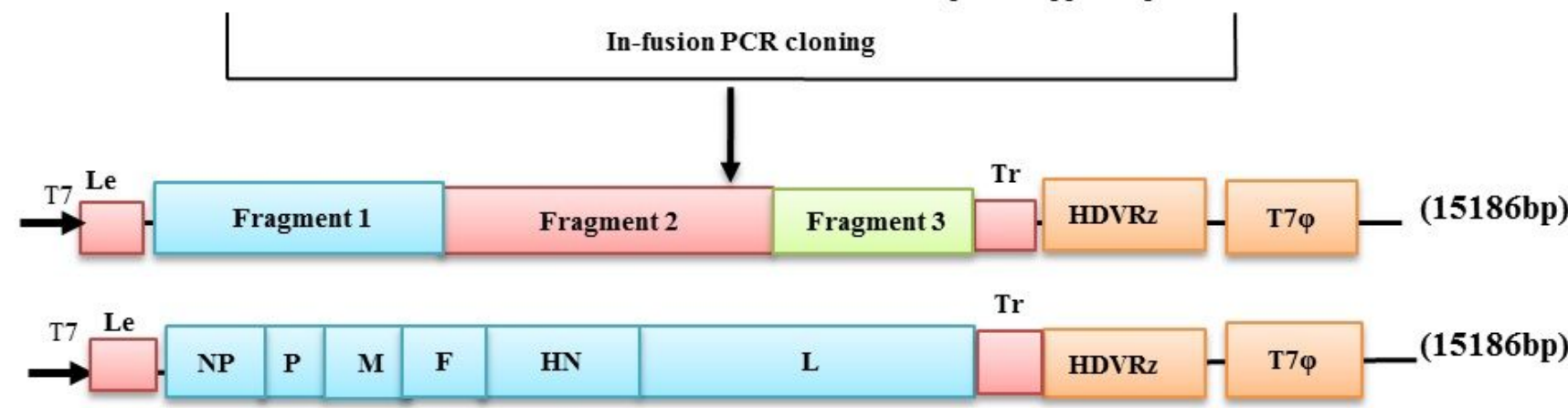

B

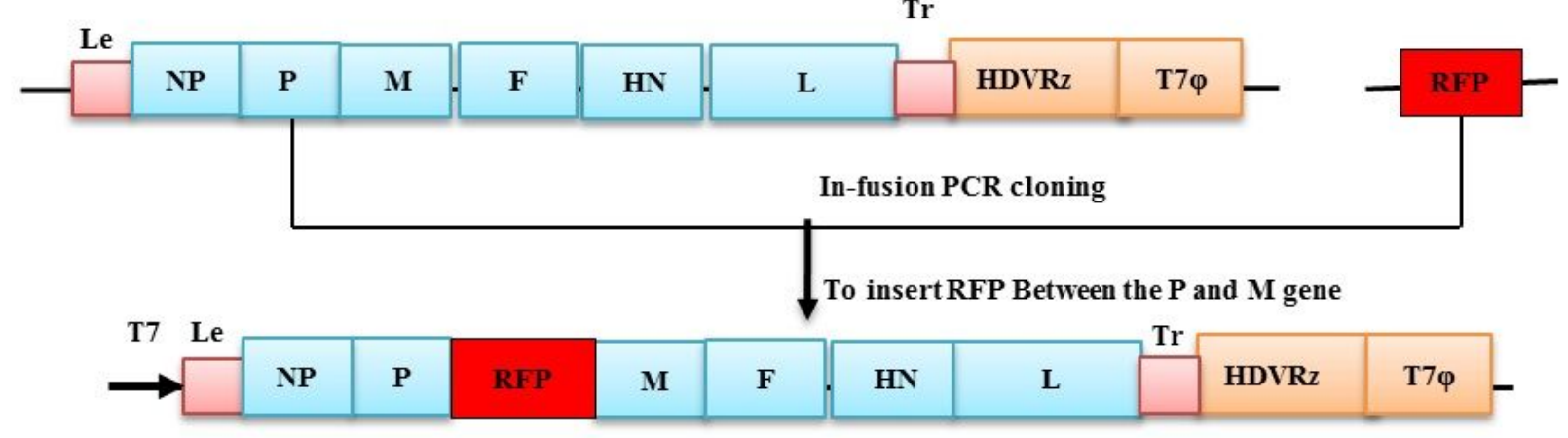

Figure 1

Schematic representation of the construction of full-length cDNA clone of NDV ZM10 strain and rZM10 expressing the RFP gene. (A) The full-length cDNA plasmid of the NDV genome was assembled from three fragments by ligation-independent cloning method. All the three fragments share $15 \mathrm{bp}$ homologous sequence and are shown by different colors: blue (Fragment 1), Red (Fragment 2) and Green (Fragment 3). The full-length clone is under the control of T7 promoter; the fragment $\mathrm{Tr}$ was followed by the hepatitis delta virus (HDV) ribozyme and the T7 terminator. (B) Cloning strategy to incorporate the RFP gene into the full length NDV clone of strain ZM10. The RFP gene was amplified from pDsRed-C1 vector and sub-cloned into the NDV full-length genome between the $\mathrm{P}$ and $\mathrm{M}$ gene as an independent transcription unit (ITU) using the In-Fusion ${ }^{\circledR}$ PCR Cloning Kit following the manufacturer's instruction (Clontech). 


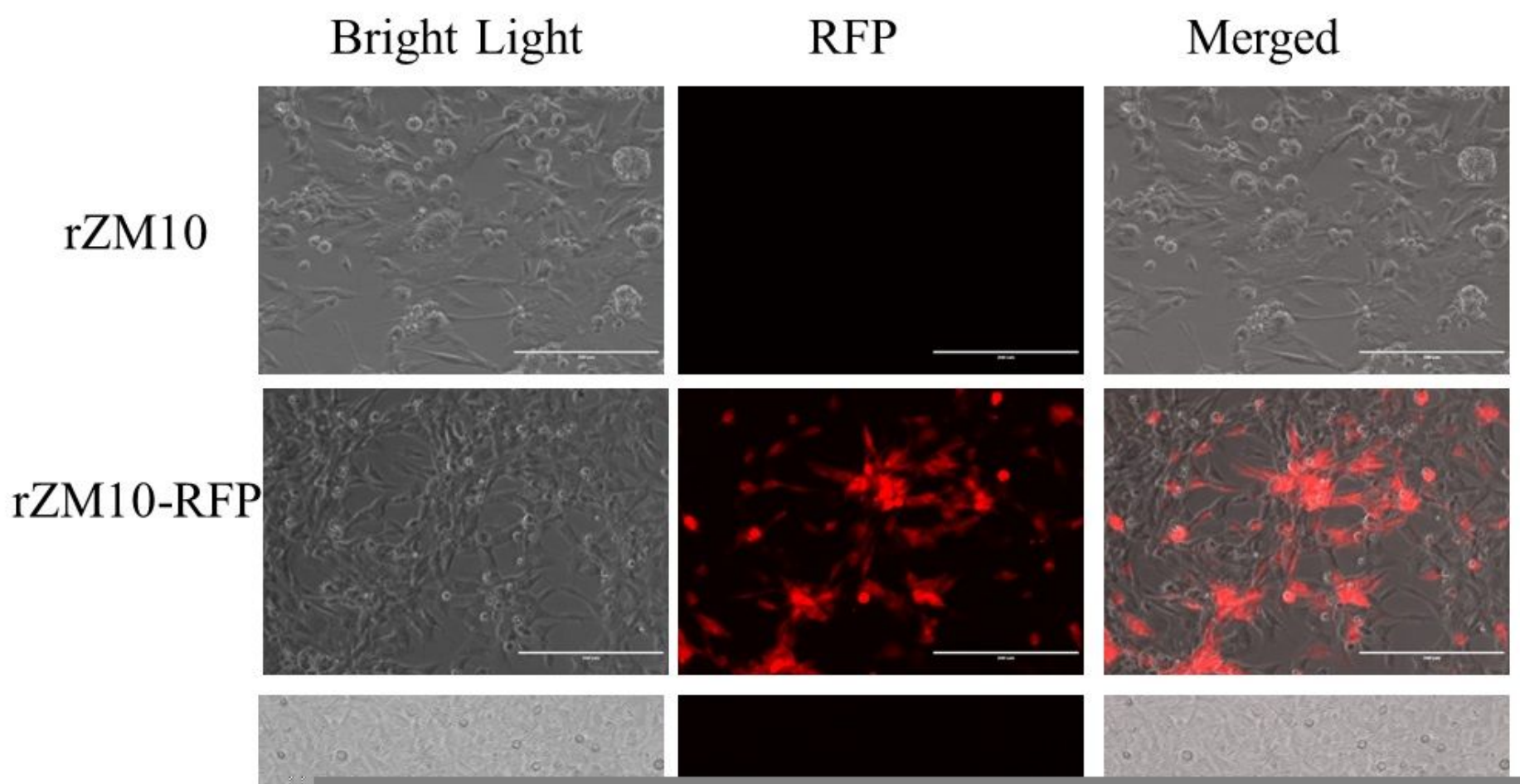

\section{Figure 2}

The CPEs and expression of RFP by the recombinant viruses rZM10 and rZM10-RFP. DF-1 cells in a 6-well plate were infected with the recombinant viruses at 0.01 m.o.i. the CPEs and the fluorescence of the infected cells were examined and digitally photographed $48 \mathrm{~h}$ post-infection, under an inverted fluorescence microscope at 6100 magnification, The DF-1 cells which were not infected was set up as a negative control. 


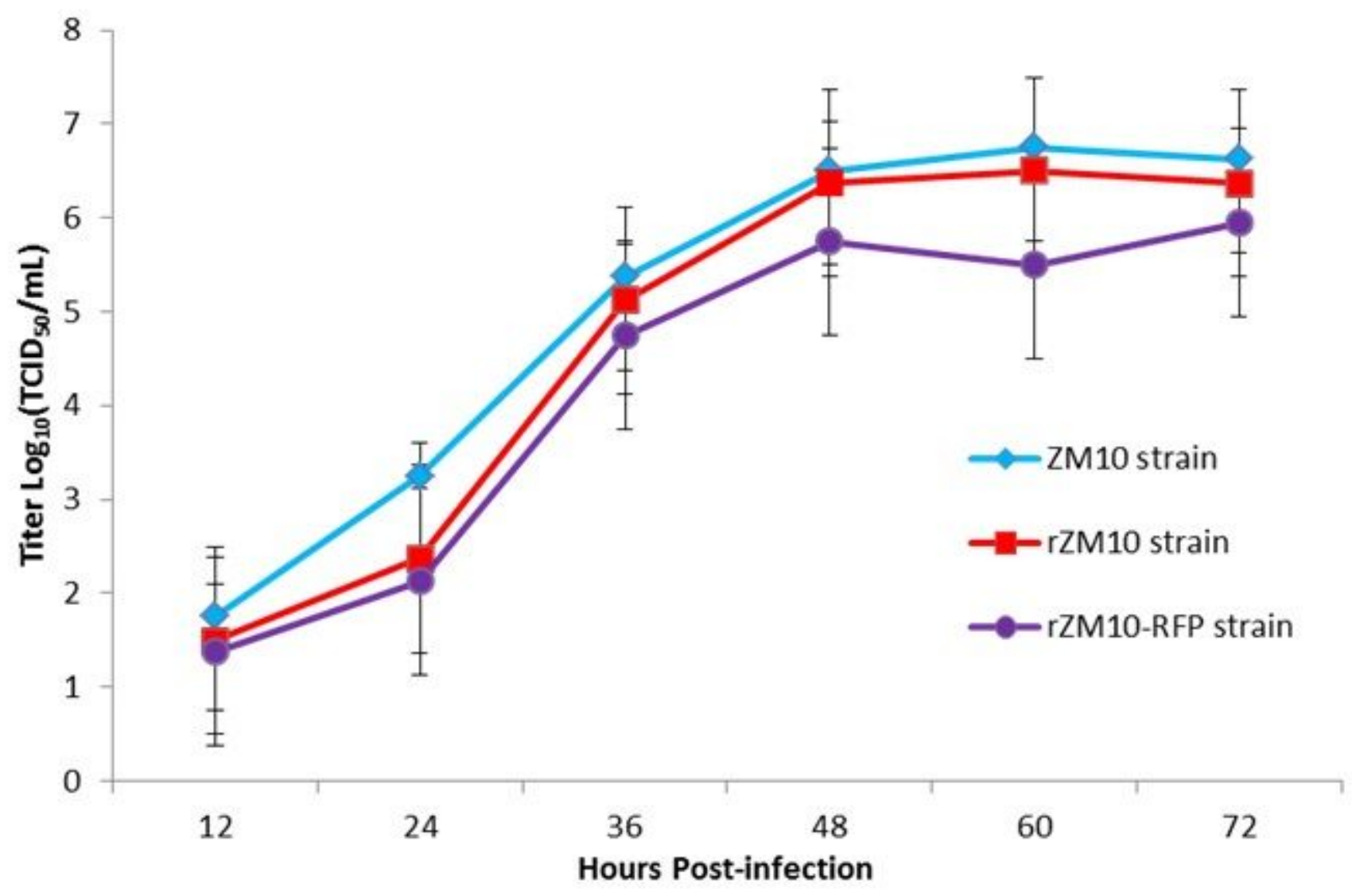

Figure 3

Growth kinetics of the recombinant viruses rZM10 and rZM10-RFP. DF-1 cells were infected with the indicated NDV viruses at $0.01 \mathrm{MOI}$. Every $12 \mathrm{~h}$ post-infection, virus lysates were harvested. Virus titers were measured by TCID 50 titration on DF- 1 cells for each time point in triplicates from two independent experiments and expressed in mean $\log 10 \mathrm{TCID} 50 / \mathrm{mL}$ with a deviation. 